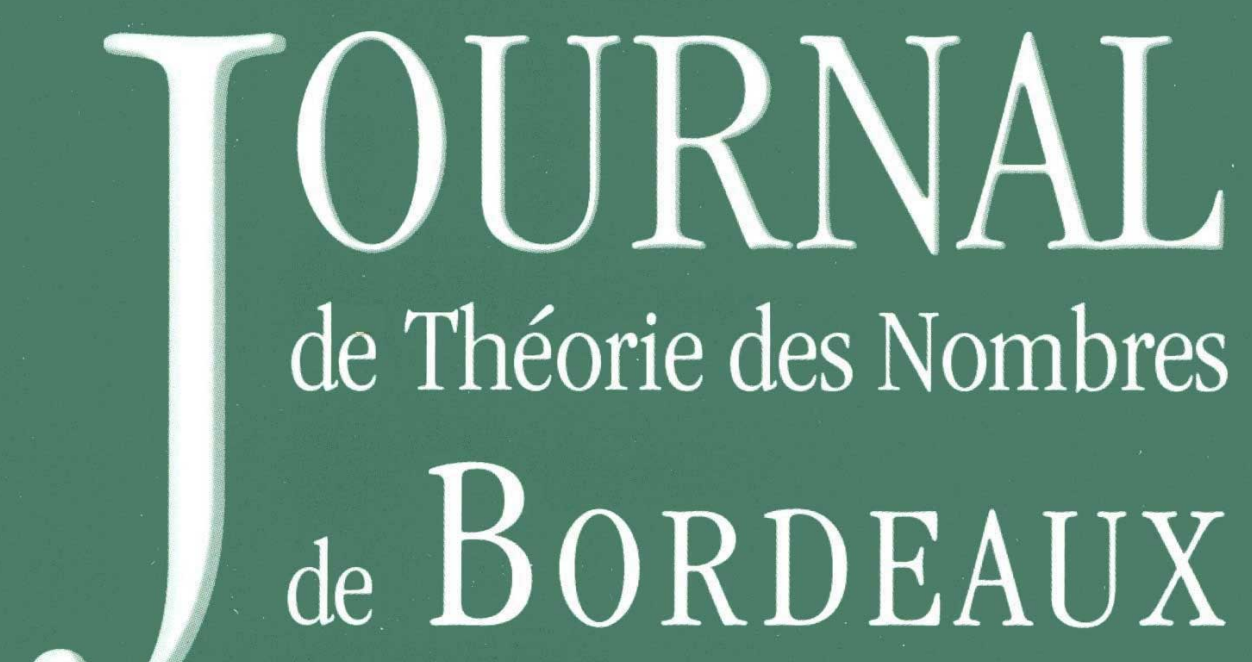

anciennement Séminaire de Théorie des Nombres de Bordeaux

\title{
Sylla LESSENI
}

Nonsolvable nonic number fields ramified only at one small prime

Tome 18, no 3 (2006), p. 617-625.

<http://jtnb.cedram.org/item?id=JTNB_2006__18_3_617_0>

(C) Université Bordeaux 1, 2006, tous droits réservés.

L'accès aux articles de la revue «Journal de Théorie des Nombres de Bordeaux » (http://jtnb.cedram.org/), implique l'accord avec les conditions générales d'utilisation (http://jtnb.cedram. org/legal/). Toute reproduction en tout ou partie cet article sous quelque forme que ce soit pour tout usage autre que l'utilisation à fin strictement personnelle du copiste est constitutive d'une infraction pénale. Toute copie ou impression de ce fichier doit contenir la présente mention de copyright.

\section{cedram}




\title{
Nonsolvable nonic number fields ramified only at one small prime
}

\author{
par SYLLA LESSENI \\ Dedicated to Michael Pohst, with best wishes \\ in the occasion of his sixtieth birthday.
}

RÉSumÉ. On montre qu'il n'existe pas de corps de nombres primitif de degré 9 ramifié en un unique premier petit. Il n'existe donc pas de corps de nombres de degré 9 ramifié en un unique premier petit et ayant un groupe de Galois non résoluble.

ABSTRACT. We prove that there is no primitive nonic number field ramified only at one small prime. So there is no nonic number field ramified only at one small prime and with a nonsolvable Galois group.

\section{Introduction}

Serre's conjecture [14] (p. 226 to 234) predicts the nonexistence of certain nonsolvable number fields which are unramified outside one small prime $p$. This has been proved by J. Tate [16] for $p=2$ by using discriminant bounding techniques. Later Serre [14] (p. 710) showed that Tate's argument could be extended to the case $p=3$ with the same conclusion.

Contrary to the works of Serre and Tate, B. Gross [7] has conjectured the existence of nonsolvable number fields ramified at exatcly one prime $p<11$. This paper follows the previous [9] which studies these fields with Galois group inside $S_{8}$. Here we will show that there are no such number fields in degree nine. As in [9], we use the method of J. Jones [8] and S. Brueggeman [1] who studied all such fields with Galois group inside $S_{6}$ and $S_{7}$ respectively.

In order to minimize the number of polynomials to be studied, we used, on one hand, methods issuing from the geometry of numbers [11] and on the other, the method developed by Odlyzko, Poitou and Serre [13] for the determination of lower bounds for discriminants.

Manuscrit reçu le 23 juin 2005.

Mots clefs. Nonic field. Galois group. Nonsolvable. 
For degree nine, the minima for discriminants are only known for totally real signature [15]. We restrict our search to all primitive number fields (see section 3) generated by an irreducible degree nine monic polynomial and which are ramified at only one prime less than 11 . To eliminate number fields only ramified at 5, we use discriminant bounding techniques depending on GRH. And then we apply another method which is unconditionally the computer search. For the rest of primes we search for the degree nine polynomials with a 2-power field discriminant, a 3-power field discriminant and those with the 7-power discriminant. The result at the end shows that only the ramification at 3 is possible, and also shows that the Galois groups inside $S_{9}$ of all such fields are solvable.

Section 2 describes theoretical aspects of ramification. We discuss the bounds on the coefficients of the polynomials generating the number fields in section 3. In the final section, we present our computer search results.

\section{Ramification at the prime $p$}

2.1. Discriminant lower and upper bounds. K. Takeuchi established in [15] the first minima for discriminants of totally real nonic number fields. He has computed the minimum for discriminants of totally real signature, and its value is 9685993193 . For the other signatures, all minimum known up to date are those concerned with the imprimitive nonic number fields containing a cubic subfield [3]. Diaz Y Diaz [4] established that a lower bound for degree nine number fields is 23007468. So the absolute value of the discriminant in degree nine cannot be less than this value.

Our main theorem given by Ore [17] on the discriminant upper bound of a number field ramified at a prime $p$ is the following:

Theorem 2.1. Let $K$ be a number field of degree $n$ and $d_{K}$ its discriminant. Let $p$ be a prime dividing $d_{K}$ and let $e_{\wp}\left(\right.$ resp. $\left.f_{\wp}\right)$ be the ramification index (resp. the inertia degree) of a prime ideal $\wp$ lying above $p$. Let $n=\sum_{i=0}^{q} b_{i} p^{i}$ $\left(0 \leq b_{i}<p\right.$ and $\left.b_{q} \neq 0\right)$ be the $p$-adic representation of $n$. Then

$i)$ the maximal possible valuation of $d_{K}$ in prime $p$ is:

$$
N_{n, p}=\sum_{i=0}^{q} b_{i}(i+1) p^{i}-h,
$$

where $h$ is the number of the coefficients $b_{i}$ which are different from zero. ii) more precisely we have:

$$
v_{p}\left(d_{K}\right) \leq \sum_{\wp \mid p} f_{\wp}\left(e_{\wp}+e_{\wp} v_{p}\left(e_{\wp}\right)-1\right) .
$$

And then $v_{p}\left(d_{K}\right)$ can assume all values from 0 to $N_{n, p}$ inclusive except $\alpha p^{\alpha}-1$ if $n=p^{\alpha}$ or if $\alpha \geq 2$ and $n=p^{\alpha}+1$. 
Butler and Mckay [2] have given in their work all the Galois groups of degree nine. And we notice that those which are nonsolvable i.e $T_{27}^{+}, T_{32}^{+}$, $T_{33}^{+}$and $T_{34}$ are primitive [5]. So in section 3 , our search for number fields will be on those which are primitive.

Throughout the paper when the context is clear, $K=\mathbb{Q}(\theta)$ will denote a nonic field where $\theta$ is a root of an irreducible degree nine monic polynomial and $L$ will denote a fixed Galois closure. Its ring of integers is denoted by $\mathbb{Z}_{K}$ and its discriminant by $d_{K}$. The discriminant of $L$ is denoted $d_{L}$. First we eliminate as many cases as possible by discriminant bounding arguments on either the nonic field $K$ or its Galois closure $L$.

2.2. Discriminant bounding arguments. The number field $K$ and its Galois closure $L$ are ramified (resp. wildly ramified) at the same single prime $p$. For more informations about it and the proofs of the following results, see [10].

Proposition 2.2. If $L$ is ramified only at 2 , the possible prime ideal decompositions of the prime 2 in $K$ is $2 \mathbb{Z}_{K}=\wp_{1}^{8} \wp_{2}$. Moreover the discriminant $d_{K}$ takes its values among $\left\{ \pm 2^{25}, \pm 2^{26}, \pm 2^{27}, \pm 2^{28}, \pm 2^{29}, \pm 2^{30}, \pm 2^{31}\right\}$.

Proof. If $K$ is tamely ramified at 2 , then by (1) we obtain $v_{2}\left(d_{K}\right) \leq 8$. Hence $\left|d_{K}\right| \leq 2^{8}$ which is less than 23007468; this case is impossible.

So if $K$ is ramified at 2 then it is wildly ramified. We get the prime ideal decompositions result by studying the different decompositions $2 \mathbb{Z}_{K}=$ $\prod_{\wp \mid 2} \wp^{e_{\wp}}$ which give the largest values of $v_{2}\left(d_{K}\right)$. Using $\left.i i\right)$ of theorem 2.1 and the discriminant lower bound given by Diaz y Diaz, we obtain the only one ramification structure. We obtain the different values of the discriminant by the fact that the minimal absolute discriminant is greater than $2^{24}$, and by theorem 2.1 which gives $v_{2}\left(d_{K}\right) \leq 31$.

Assuming that $L$ is ramified only at 2, the following proposition is essential to eliminate $T_{27}^{+}$and $T_{32}^{+}$as Galois groups inside $S_{9}$.

Proposition 2.3. If the Galois closure $L$ of $K$ is ramified only at 2 then its Galois group is not isomorphic to $T_{27}^{+}$nor $T_{32}^{+}$.

Proof. Modifying (1) for Galois extension yields $v_{2}\left(d_{L}\right) \leq|G|\left(1+v_{2}(e)-\right.$ $1 / e)$. Hence $\left|d_{L}\right|^{1 /|G|} \leq 2^{\left(1+v_{2}(e)-1 / e\right)}$. We obtain $\left|d_{L}\right|^{1 / 504} \leq 15.978$ for $G=T_{27}^{+}$(resp. $\left|d_{L}\right|^{1 / 1512} \leq 15.993$ for $G=T_{32}^{+}$). On the other hand, using the lower bound of the root discriminant in [4], we obtain $\left|d_{L}\right|^{1 / 504} \geq 20.114$ (resp. $\left|d_{L}\right|^{1 / 1512} \geq 21.253$ ). This is a contradiction.

Proposition 2.4. If $L$ is ramified only at 3 , the possible decompositions of $p=3$ in $\mathbb{Z}_{K}$ are $3 \mathbb{Z}_{K}=\wp^{9}$ or $3 \mathbb{Z}_{K}=\wp_{1}^{6} \wp_{2}^{3}$. Moreover the discriminant $d_{K}$ takes its values among $\left\{3^{16}, 3^{18},-3^{19}, 3^{20},-3^{21}, 3^{22},-3^{23}, 3^{24},-3^{25}, 3^{26}\right\}$. 
Proof. Use the method developed in the proof of Proposition 2.2. Then we apply the Stickelberger identity, $d_{K} \equiv 0,1(\bmod 4)$ to have the sign of the discriminant.

Remark. By using unconditionally in [13] the local corrections corresponding to small prime numbers, we can eliminate the values $d_{K}= \pm 2^{25}$, $d_{K}= \pm 2^{26}$ and $d_{K}=3^{16}$ of the discriminant. And we also show that the decomposition $3 \mathbb{Z}_{K}=\wp_{1}^{6} \wp_{2}^{3}$ is not possible.

Proposition 2.5. a) Assuming GRH, the Galois closure $L$ of $K$ cannot be ramified only at 5 .

b) If GRH does not hold, the possible ramification structures at $p=5$ in $K$ are $5 \mathbb{Z}_{K}=\wp_{1}^{5} \wp_{2}^{4}, 5 \mathbb{Z}_{K}=\wp_{1}^{5} \wp_{2}^{3} \wp_{3}, 5 \mathbb{Z}_{K}=\wp_{1}^{5} \wp_{2}^{2} \wp_{3}^{2}$ or $5 \mathbb{Z}_{K}=\wp_{1}^{5} \wp_{2}^{2}$ with inertia degree $f_{\wp_{2}}=2$ in this last case. And then the discriminant of $K$ is $d_{K}=5^{11}$ or $d_{K}=5^{12}$.

Proof. a) If $L$ is tamely ramified at 5 , then $K$ is also tamely ramified at 5 and by $(1)$ we have $v_{5}\left(d_{K}\right) \leq 8$. Hence $\left|d_{K}\right|$ would be less than 23007468 in this case. This is a contradiction.

Now suppose $L$ is wildly ramified at 5 and let $G$ be its Galois group. We show that 45 divides $|G|$, and so $G$ is $T_{34}$ or $T_{33}^{+}$. Let $d_{L}$ be the discriminant of $L$ and let $e$ be the ramification index of a chosen prime ideal $\mathfrak{P}$ lying over 5 in the ring of integers of $L$. Also, the inertia subgroup, denoted $G_{0}$, has order $e$. Since the size of the Galois group $G$ is divisible by 5 and not by 25 , this implies that $v_{5}(e)=1$. So the wild inertia subgroup $G_{1}$ has order 5 . The normalizer of a 5-cycle has order 480 in $S_{9}$ and 240 in $A_{9}$. Since $G_{0} / G_{1}$ is cyclic, we show that $e$ cannot be 480 ; and so $e$ divides 240. Modifying (1) of theorem 2.1 for a Galois extension yields $v_{5}\left(d_{L}\right) \leq f(e+e-1) g \leq|G|(2-1 / e)$. Hence $\left|d_{L}\right|^{1 /|G|} \leq 5^{\frac{479}{240}} \approx 24.833$.

On the other hand, the GRH implies that we have Poitou's following inequality [1]

$$
\frac{1}{|G|} \log \left|d_{L}\right| \geq\left(3.801-\frac{20.766}{(\log |G|)^{2}}-\frac{157.914(1+1 /|G|)}{(\log |G|)^{3}\left(1+\frac{\pi^{2}}{(\log |G|)^{2}}\right)^{2}}\right) .
$$

We obtain $\left|d_{L}\right|^{1 /\left|T_{34}\right|} \geq 36.22$ and $\left|d_{L}\right|^{1 /\left|T_{33}^{+}\right|} \geq 35.09$. This a contradiction. b) Use the technique developed in the proof of Proposition 2.2.

Let move now to the last case i.e for $p=7$.

Proposition 2.6. If $L$ is ramified only at 7 , the possible decompositions of $p=7$ in $K$ are $7 \mathbb{Z}_{K}=\wp_{1}^{7} \wp_{2}^{2}$ or $7 \mathbb{Z}_{K}=\wp_{1}^{7} \wp_{2} \wp_{3}$ or $7 \mathbb{Z}_{K}=\wp_{1}^{7} \wp_{2}$ with inertia degree $f_{2}=2$ in the last case. Moreover the discriminant $d_{K}$ takes its values among $\left\{7^{8},-7^{9}, 7^{10},-7^{11}, 7^{12},-7^{13}, 7^{14}\right\}$.

Proof. If $K$ is ramified at only 7 , then it is wildly ramified. Using theorem 2.1 , we have $v_{7}\left(d_{K}\right) \leq 14$. Then we apply the Stickelberger identity and the fact that $\left|d_{K}\right| \geq 23007468$. 


\section{Polynomials generating the nonic number fields}

We have shown in the previous section that the set of nonic number fields $K$ can be restricted to those which are primitive. For the rest of the paper $K$ will be considered primitive and $p$ will be the prime $2,3,5$ or 7 .

3.1. Notation. We use here the notations of [1]. Let $I$ be the product of all prime ideals in $\mathbb{Z}_{K}$ above primes dividing the discriminant $d_{K}$ of $K$. Each $\theta \in I \backslash \mathbb{Z}$ has a minimal polynomial $f_{\theta}(x)$ in $\mathbb{Z}[x]$ of the form $f_{\theta}(x)=x^{9}+a_{1} x^{8}+a_{2} x^{7}+a_{3} x^{6}+a_{4} x^{5}+a_{5} x^{4}+a_{6} x^{3}+a_{7} x^{2}+a_{8} x+a_{9}$. It will be sufficient to search for polynomials having a root contained in $I$. We need the quadratic form $\mathcal{T}_{2}=\mathcal{T}_{2}(\theta)=\sum_{i=1}^{n}\left|\theta_{i}\right|^{2}$ in the roots of $f_{\theta},(\theta)_{i}$ where $1 \leq i \leq 9$.

We must reduce the search set of polynomials to a finite set. The coefficients $a_{i}$ of $f_{\theta}$ are restricted by the quadratic form $\mathcal{T}_{2}$. We use a version of Hunter's theorem [8] adapted to this context by Jones and Roberts (see below). It guarantees the existence of one $\theta \in I \backslash \mathbb{Z}$ with the corresponding coefficients $a_{i}$ satisfying the congruence $p^{\alpha_{i}} \mid a_{i}$, where $\alpha_{i}$ is a positive integer.

Theorem 3.1 (Jones et Roberts 1999). Let $K$ be a degree $n \geq 3$, primitive number field, with discriminant $d_{K}$. Let $l$ be the least positive integer contained in I and let $m$ be the order of $\mathbb{Z}_{K} / I$. Finally, let $\gamma_{n}$ be Hermite's constant of $n$-dimensional lattices. Then there exists an element $\theta \in I \backslash \mathbb{Z}$ such that

i) $\mathcal{T}_{2}(\theta) \leq \frac{a_{1}^{2}}{n}+\gamma_{n-1}\left(\frac{m^{2}\left|d_{K}\right|}{l^{2} n}\right)^{1 / n-1}$

ii) $0 \leq a_{1} \leq n . l / 2$.

3.2. Newton-Ore exponents. Jones and Roberts define a Newton-Ore exponent, $\alpha_{i}$, to be the largest integer such that $p^{\alpha_{i}}$ divides $a_{i}$ for all polynomial $f_{\theta}$ with $\theta$ in the search ideal $I$. We search for the required minimal power of the prime $p$ to guarantee that the polynomial discriminant is divided by a power of $p$. See the following tables.

In the totally ramified case, we notice that $p$ divides the constant term $a_{9}$. We find the required power of $p$ for the other $a_{i}$ by using the fact that if $\pi$ is a uniformizer with polynomial $F(x)$, then the different is generated by $F^{\prime}(\pi)$. Details are given in [11]. For the other ramification structures, we use the method described in [8].

Corollary 3.2. Let $K$ be a degree 9 , primitive number field, with absolute discriminant $2^{r}$. Then there exists an element $\theta \in I \backslash \mathbb{Z}$ such that

1) $\mathcal{T}_{2}(\theta) \leq U_{2}=\frac{a_{1}^{2}}{9}+\left(\frac{2^{10+r}}{9}\right)^{1 / 8}$

2) $a_{1}=0,2,4,6$ or 8 . 
TABLE 1

Newton-Ore exponents for the different ramification structures at $p$.

\begin{tabular}{|c|c|c|c|c|c|c|c|c|c|c|}
\hline$d_{K}$ & $a_{1}$ & $a_{2}$ & $a_{3}$ & $a_{4}$ & $a_{5}$ & $a_{6}$ & $a_{7}$ & $a_{8}$ & $a_{9}$ \\
\hline $\pm 2^{31}$ & 1 & 3 & 4 & 2 & 3 & 3 & 4 & 1 & 2 \\
\hline $\pm 2^{30}$ & 1 & 3 & 4 & 2 & 3 & 3 & 4 & 1 & 2 \\
\hline $\pm 2^{29}$ & 1 & 2 & 3 & 2 & 3 & 3 & 4 & 1 & 2 \\
\hline $\pm 2^{28}$ & 1 & 2 & 3 & 2 & 3 & 3 & 4 & 1 & 2 \\
\hline $\pm 2^{27}$ & 1 & 2 & 3 & 1 & 2 & 3 & 4 & 1 & 2 \\
\hline${ }^{8} \wp_{2}$ & $a_{1}$ & $a_{2}$ & $a_{3}$ & $a_{4}$ & $a_{5}$ & $a_{6}$ & $a_{7}$ & $a_{8}$ & $a_{9}$ \\
\hline $3_{K}{ }^{26}$ & 3 & 3 & 2 & 3 & 3 & 2 & 3 & 3 & 1 \\
\hline$-3^{25}$ & 2 & 3 & 2 & 3 & 3 & 2 & 3 & 3 & 1 \\
\hline $3^{24}$ & 2 & 2 & 2 & 3 & 3 & 2 & 3 & 3 & 1 \\
\hline$-3^{23}$ & 2 & 2 & 1 & 3 & 3 & 2 & 3 & 3 & 1 \\
\hline $3^{22}$ & 2 & 2 & 1 & 2 & 3 & 2 & 3 & 3 & 1 \\
\hline$-3^{21}$ & 2 & 2 & 1 & 2 & 2 & 2 & 3 & 3 & 1 \\
\hline $3^{20}$ & 2 & 2 & 1 & 2 & 2 & 1 & 3 & 3 & 1 \\
\hline$-3^{19}$ & 2 & 2 & 1 & 2 & 2 & 1 & 2 & 3 & 1 \\
\hline $3^{18}$ & 2 & 2 & 1 & 2 & 2 & 1 & 2 & 2 & 1 \\
\hline
\end{tabular}

Corollary 3.3. Let $K$ be a degree 9, primitive number field, with absolute discriminant $3^{s}$. Then there exists an element $\theta \in I \backslash \mathbb{Z}$ such that 1) $\mathcal{T}_{2}(\theta) \leq U_{2}=\frac{a_{1}^{2}}{9}+2 \times 3^{\frac{s-2}{8}}$.

2) If $d_{K}=3^{26}$ then $a_{1}=0$. If $d_{K} \in\left\{3^{18},-3^{19}, 3^{20},-3^{21}, 3^{22},-3^{23}, 3^{24}\right.$, $\left.-3^{25}\right\}$ then $a_{1}=0$ or $a_{1}=9$.

$$
5 \mathbb{Z}_{K}=\wp_{1}^{5} \wp_{2}^{4}
$$

\begin{tabular}{|c|c|c|c|c|c|c|c|c|c|}
\hline$d_{K}$ & $a_{1}$ & $a_{2}$ & $a_{3}$ & $a_{4}$ & $a_{5}$ & $a_{6}$ & $a_{7}$ & $a_{8}$ & $a_{9}$ \\
\hline $5^{12}$ & 1 & 1 & 1 & 1 & 1 & 2 & 2 & 2 & 2 \\
\hline $5^{11}$ & 1 & 1 & 1 & 1 & 1 & 2 & 2 & 2 & 2 \\
\hline
\end{tabular}

$$
5 \mathbb{Z}_{K}=\wp_{1}^{5} \wp_{2}^{3} \wp_{3}
$$

\begin{tabular}{|c|c|c|c|c|c|c|c|c|c|}
\hline$d_{K}$ & $a_{1}$ & $a_{2}$ & $a_{3}$ & $a_{4}$ & $a_{5}$ & $a_{6}$ & $a_{7}$ & $a_{8}$ & $a_{9}$ \\
\hline $5^{11}$ & 1 & 1 & 1 & 2 & 1 & 2 & 2 & 2 & 3 \\
\hline
\end{tabular}

$$
\begin{aligned}
& 5 \mathbb{Z}_{K}=\wp_{1}^{5} \wp_{2}^{2} \wp_{3}^{2} \quad \text { or } \\
& 5 \mathbb{Z}_{K}=\wp_{1}^{5} \wp_{2}^{2}
\end{aligned}
$$

\begin{tabular}{|c|c|c|c|c|c|c|c|c|c|}
\hline$d_{K}$ & $a_{1}$ & $a_{2}$ & $a_{3}$ & $a_{4}$ & $a_{5}$ & $a_{6}$ & $a_{7}$ & $a_{8}$ & $a_{9}$ \\
\hline $5^{11}$ & 1 & 1 & 2 & 2 & 1 & 2 & 2 & 3 & 3 \\
\hline
\end{tabular}


When GRH does not hold, we have given the different ramification structures at 5 . We show also that there exists an element $\theta \in I \backslash \mathbb{Z}$ such that its trace $a_{1}=0,5,10,15$ or 20 .

$7 \mathbb{Z}_{K}=\wp_{1}^{7} \wp_{2}^{2}$

\begin{tabular}{|c|c|c|c|c|c|c|c|c|c|}
\hline$d_{K}$ & $a_{1}$ & $a_{2}$ & $a_{3}$ & $a_{4}$ & $a_{5}$ & $a_{6}$ & $a_{7}$ & $a_{8}$ & $a_{9}$ \\
\hline $7^{14}$ & 1 & 1 & 2 & 2 & 2 & 2 & 1 & 2 & 2 \\
\hline$-7^{13}$ & 1 & 1 & 2 & 2 & 2 & 2 & 1 & 2 & 2 \\
\hline $7^{12}$ & 1 & 1 & 2 & 2 & 2 & 2 & 1 & 2 & 2 \\
\hline$-7^{11}$ & 1 & 1 & 1 & 2 & 2 & 2 & 1 & 2 & 2 \\
\hline $7^{10}$ & 1 & 1 & 1 & 1 & 2 & 2 & 1 & 2 & 2 \\
\hline$-7^{9}$ & 1 & 1 & 1 & 1 & 1 & 2 & 1 & 2 & 2 \\
\hline
\end{tabular}

$7 \mathbb{Z}_{K}=\wp_{1}^{7} \wp_{2} \wp_{3}$

or

$7 \mathbb{Z}_{K}=\wp_{1}^{7} \wp_{2}$

\begin{tabular}{|c|c|c|c|c|c|c|c|c|c|}
\hline$d_{K}$ & $a_{1}$ & $a_{2}$ & $a_{3}$ & $a_{4}$ & $a_{5}$ & $a_{6}$ & $a_{7}$ & $a_{8}$ & $a_{9}$ \\
\hline$-7^{13}$ & 1 & 2 & 2 & 2 & 2 & 2 & 1 & 2 & 3 \\
\hline $7^{12}$ & 1 & 2 & 2 & 2 & 2 & 2 & 1 & 2 & 3 \\
\hline$-7^{11}$ & 1 & 1 & 2 & 2 & 2 & 2 & 1 & 2 & 3 \\
\hline $7^{10}$ & 1 & 1 & 1 & 2 & 2 & 2 & 1 & 2 & 3 \\
\hline$-7^{9}$ & 1 & 1 & 1 & 1 & 2 & 2 & 1 & 2 & 3 \\
\hline
\end{tabular}

Corollary 3.4. Let $K$ be a degree 9, primitive number field, with absolute discriminant $7^{c}$. Then there exists an element $\theta \in I \backslash \mathbb{Z}$ such that

1) i) If $7 \mathbb{Z}_{K}=\wp_{1}^{7} \wp_{2}^{2}$ then $\mathcal{T}_{2}(\theta) \leq U_{2}=\frac{a_{1}^{2}}{9}+2 \times\left(\frac{7^{c+2}}{9}\right)^{1 / 8}$

ii) If $7 \mathbb{Z}_{K}=\wp_{1}^{7} \wp_{2} \wp_{3}$ or $7 \mathbb{Z}_{K}=\wp_{1}^{7} \wp_{2}$ then $\mathcal{T}_{2}(\theta) \leq U_{2}=\frac{a_{1}^{2}}{9}+2 \times\left(\frac{7^{c+4}}{9}\right)^{1 / 8}$.

2) $a_{1} \in\{0,7,14,21,28\}$.

3.3. Coefficients bounds. The bounds on $a_{1}$ were discussed previously. We use the method developed by M. Pohst in [12] and Newton-Ore exponents to give the values of the other coefficients $a_{i}$ of the minimal polynomial $f_{\theta}$. Bounding $f_{\theta}( \pm 1)$, we obtain better bounds on $a_{6}$ and $a_{7}$ by using the fact that $a_{6}=\frac{f_{\theta}(1)-f_{\theta}(-1)}{2}-\left(1+a_{1}+a_{2}+a_{4}+a_{8}\right)$ and $a_{7}=$ $\frac{f_{\theta}(1)+f_{\theta}(-1)}{2}-\left(a_{1}+a_{3}+a_{5}+a_{9}\right)$.

We can improve the results in [4] by using unconditionally the local corrections corresponding to small prime numbers for all signatures of nonic number fields. With the results given in [13] we can eliminate much values of the constant term $a_{9}$ and discriminants $d_{K}$ because of the signature. 


\section{Computer search results}

In this section, we explain in more detail, how one can make much quicker searches for primitive nonic fields with $p$-power discriminant, where $p=$ $2,3,5$ or 7 . The program we use for these searches is written in $\mathrm{C}$, using the Pari programming library [6].

Fixing the signature for the first stage, we eliminate over half the polynomials. Then using the relation $d_{f_{\theta}}=d_{K} a^{2}$, where $d_{f_{\theta}}$ is the discriminant of $f_{\theta}$, we discard all but finitely many polynomials because of the valuation at the single prime $p$. We check the few remaining polynomials for irreducibility: most of them are irreducible. In the final stage, we compute the field discriminants: no polynomial is found with 2-power, 5-power or 7-power field discriminant. For the polynomials with 3-power field discriminant, we determine the Galois group and a minimal polynomial which generates the same field by 'polgalois' and 'polredabs' commands in [6].

TABLE 2

Search results with $d_{K}$ of the form $\pm 3^{s}$.

\begin{tabular}{|c|l|c|c|}
\hline$s$ & polynomials $f_{\theta}(x)$ & signature & Gal $(\mathrm{L} / \mathbb{Q})$ \\
\hline 19 & $x^{9}-3 x^{6}-6 x^{3}-1$ & $(3,3)$ & $T_{4}$ \\
\hline 21 & $x^{9}-3 x^{6}+1$ & $(3,3)$ & $T_{13}$ \\
22 & $x^{9}-6 x^{6}+12 x^{3}+1$ & $(1,4)$ & $T_{11}^{+}$ \\
22 & $x^{9}-9 x^{7}+27 x^{5}-30 x^{3}+9 x-1$ & $(9,0)$ & $T_{1}^{+}$ \\
\hline 23 & $x^{9}-6 x^{6}+9 x^{3}-3$ & $(3,3)$ & $T_{22}$ \\
23 & $x^{9}-3 x^{6}+3$ & $(3,3)$ & $T_{22}$ \\
23 & $x^{9}-3 x^{6}-9 x^{3}+3$ & $(3,3)$ & $T_{22}$ \\
\hline 25 & $x^{9}-9 x^{7}-3 x^{6}+27 x^{5}+18 x^{4}-24 x^{3}$ & $(3,3)$ & $T_{20}$ \\
& $-27 x^{2}-9 x+23$ & & \\
25 & $x^{9}-9 x^{7}-6 x^{6}+27 x^{5}+36 x^{4}-24 x^{3}$ & $(3,3)$ & $T_{20}$ \\
& $-54 x^{2}-9 x+22$ & & \\
25 & $x^{9}-9 x^{7}-3 x^{6}+27 x^{5}+18 x^{4}-15 x^{3}$ & $(3,3)$ & $T_{20}$ \\
& $-27 x^{2}-36 x-4$ & & \\
26 & $x^{9}-9 x^{6}+27 x^{3}-3$ & $(1,4)$ & $T_{3}^{+}$ \\
26 & $x^{9}-3$ & $(1,4)$ & $T_{10}^{+}$ \\
26 & $x^{9}-9 x^{6}+27 x^{3}-24$ & $(1,4)$ & $T_{10}^{+}$ \\
\hline
\end{tabular}

After eliminating duplicate fields, there are 13 distinct number fields ramified only at 3 . The search for primitive number fields of degree nine and 2-power, 5-power or 7-power discriminant came up empty in all cases. 
Since all of the fields found are imprimitive and so have a solvable Galois group (see the table 2), we have proved the following results.

Theorem 4.1. There is no primitive nonic number field ramified at only a single prime $p$, where $p<11$.

Corollary 4.2. Let $K$ be a nonic number field which is ramified at only a single prime $p$ and $p<11$. Then the Galois group of its Galois closure is not nonsolvable.

\section{References}

[1] S. Brueggeman, Septic Number Fields Which are Ramified Only at One Small Prime. J. Symbolic Computation 31 (2001), 549-555.

[2] G. Butler, J. MCKAy, The transitive groups of degree up to eleven. Comm. Algebra 11 (1983), no. 8, 863-911.

[3] F. Diaz Y Diaz, M. Olivier, Imprimitive ninth-degree fields with small discriminants. Math. Comp. 64 (1995), no. 209, 305-321.

[4] F. Diaz Y Diaz, Tables minorant la racine n-ième du discriminant d'un corps de nombres de degré n. Publications Mathématiques d'Orsay 80.06 (1980).

[5] Y. Eichenlaub, Problèmes effectifs de théorie de Galois en degré 8 à 11. Thèse soutenue à l'université de Bordeaux 1 en 1996.

[6] ftp://megrez.math.u-bordeaux.fr/pub/pari/

[7] B. Gross, Modular forms $(\bmod p)$ and Galois representation. Inter. Math. Res. Notices 16 (1998), 865-875.

[8] J. Jones, D. Roberts, Sextic number fields with discriminant $(-1)^{j} 2^{a} 3^{b}$. In Number Theory : Fifth Conference of the Canadian Number Theory Association, CRM Proceedings and Lecture Notes 19, 141-172. American Mathematical Society, 1999.

[9] S. Lesseni, The non-existence of nonsolvable octic number fields ramified only at one small prime. Mathematics of Computation 75 no. 255 (2006), 1519-1526.

[10] S. LeSSENI, http://www.math.unicaen.fr/ lesseni/index.html. See "ma thèse".

[11] J. Martinet, Petits discriminants des corps de nombres. In Number theory days, 1980 (Exeter, 1980), volume 56 of London Math. Soc. Lecture Note Series, 151-193. Cambridge Uni. Press, Cambridge, 1982.

[12] M. PoHst, On the computation of number fields of small discriminants including the mininum discriminants of sixth degree fields. J. Number Theory 14 (1982), 99-117.

[13] S. Selmane, Odlyzko-Poitou-Serre lower bounds for discriminants for number fields. Maghreb Math. Rev. 8 (1999), no 18.2.

[14] J. P. Serre, Oeuvres, vol. 3. Springer-Verlag, Berlin/New York, 1986.

[15] K. TAKeuchi, Totally real algebraic number fields of degree 9 with small discriminants. Saitama Math. J. 17 (1999), 63-85.

[16] J. TATE, The non-existence of certain Galois extension of $\mathbb{Q}$ unramified outside 2 . Contemp. Math. 174 (1994) 153-156.

[17] R. Thompson, On the possible forms of discriminants of algebraic fields II. American J. of Mathematics 55 (1933), 110-118.

\section{Sylla Lesseni}

Université Bordeaux 1

Laboratoire d'Algorithmique Arithmétique

351, Cours de la Libération

33405 Talence Cedex, France.

E-mail: Sylla.Lesseni@math.u-bordeaux1.fr 